\section{Outcomes of 23-gauge pars plana vitrectomy in combined scleral buckling and vitrectomy for complex rhegmatogenous retinal detachments}

\author{
Scott D. Schoenberger, \\ Daniel M. Miller, ${ }^{1,2}$ \\ Christopher D. Riemann, 1,2 \\ Robert E. Foster, ${ }^{2}$ Michael R. Petersen ${ }^{2}$ \\ 'Department of Ophthalmology, \\ University of Cincinnati; ${ }^{2}$ Cincinnati Eye \\ Institute, Cincinnati, Ohio, USA
}

\section{Abstract}

Rhegmatogenous retinal detachments associated with proliferative vitreoretinopathy, giant retinal tears, ocular trauma, proliferative diabetic retinopathy, or necrotizing retinitis are considered more complex than those without these factors. The aim of the current review is to address the surgical outcomes and complications of 23-gauge pars plana vitrectomy with scleral buckling (23GPPV/SB) for repair of these complex retinal detachments. This retrospective study involved 54 eyes of 53 patients who underwent 23GPPV/SB between July 2007 and September 2009. Preoperative diagnosis, surgical technique, preoperative and postoperative visual acuities, intraoperative and postoperative complications, and anatomic reattachment rates were examined. Fifty-four eyes of 53 patients were reviewed in this study and indications for surgery varied. Mean logarithm of the minimal angle of resolution (logMAR) pre- and post-operative visual acuities were $1.166(20 / 293)$ and 0.780 (20/120), respectively, which led to a statistically significant improvement in logMAR $(\mathrm{P}=0.0165)$. Single operation and final reattachment rates were $87 \%$ (47 of 54 eyes) and $100 \%$, respectively. Postoperative complications included choroidal effusion/hemorrhage (14.8\%, 8 of 54 eyes) and vitreous hemorrhage (11.1\%, 6 of 54 eyes). Other more infrequent complications included hyphema (9.3\%, 5 of 54 eyes), hypotony (5.6\%, 3 of 54 eyes) and ocular hypertension $>35 \mathrm{mmHg}(3.7 \%, 2$ of 54 eyes). A total of $31.5 \%$ (17 of 54 eyes) of patients had a complication in the postoperative time period, but $58.8 \%$ of these resolved spontaneously without requiring an intervention. 23GPPV/SB may be considered for complex retinal detachment repair with good anatomic reattachment rates, but with relatively high complication rates.

\section{Introduction}

Advancements in surgical instrumentation have led to changes in the surgical repair of rhegmatogenous retinal detachments (RRDs). Key components of RRD repair include pars plana vitrectomy (PPV), scleral buckling procedure (SBP), intraocular gas and silicone oil (S0) infusion, endolaser photocoagulation, perfluorocarbon liquids, and wide-angle viewing systems. ${ }^{1-4}$ The evolution of smaller gauge vitrectomy instruments has been a significant technological advance in vitreoretinal surgery. There has been a trend towards the use of smaller gauge vitrectomy instruments to treat increasing complex posterior segment pathology. ${ }^{5-7}$ Twenty three- and 25-gauge instruments have been used in primary pseudophakic retinal detachment repair. ${ }^{8}$ There have also been reports of their use in more complex retinal detachments requiring silicone oil.9,10

There is a spectrum of pathology in the treatment of RRDs. Generally, RRDs associated with proliferative vitreoretinopathy (PVR), giant retinal tears (GRT), ocular trauma, proliferative diabetic retinopathy (PDR), or necrotizing retinitis are considered more complex than RRDs without these factors. ${ }^{11}$ Many vitreoretinal surgeons would manage these more complex cases utilizing 20-gauge pars plana vitrectomy, and in many instances, with combined scleral buckling. ${ }^{12-18}$ However, the management of complex RRDs varies by surgeon preference and experience.

The aim of this retrospective study was to examine the anatomic success, visual outcomes, and complications of patients with complex RRDs treated with 23-gauge pars plana vitrectomy with scleral buckling (23GPPV/SB). Indications for surgery in this review included RRDs associated with PVR (any grade), GRT, ocular trauma, high myopia and multiple tears.

\section{Materials and Methods}

Patients who underwent 23-gauge PPV with SBP between July 2007 and September of 2009 by one of four surgeons at the Cincinnati Eye Institute were retrospectively identified. All patients provided preoperative informed consent for surgery. After the final follow up visit for each patient, data were retrospectively reviewed and were collected in accordance with compliance guidelines set forth by the Health Insurance Portability and Accountability Act of 1996. Institutional review board (IRB) approval was obtained from the University of Cincinnati IRB prior to data collection. All patients undergoing 23GPPV/SB between the time frames mentioned above
Correspondence: Daniel M. Miller, 1945 CEI Drive, Cincinnati, OH 45242, USA.

Tel. +1.513.984.5133 - Fax: +1.513.984.2390 Email: dmiller@cincinnatieye.com

Key words: pars plana vitrectomy, scleral buckling, complex rhegmatogenous retinal detachment.

Conflict of interest: the authors report no conflicts of interest.

Contributions: all the authors contributed equally

Received for publication: 17 April 2011.

Accepted for publication: 24 June 2011.

This work is licensed under a Creative Commons Attribution NonCommercial 3.0 License (CC BYNC 3.0).

○Copyright S.D. Schoenberger et al., 2011 Licensee PAGEPress, Italy

Eye Reports 2011; 1:e3

doi:10.4081/eye.2011.e3

with at least one month of follow-up were included in the study.

All patients underwent either local anesthesia with monitored anesthesia care and retrobulbar anesthesia or general anesthesia. The periocular skin was prepared with $5 \%$ povidone-iodine followed by a drop of povidone-iodine in the inferior fornix. The eye was prepared and draped in standard fashion and a lid speculum was placed. A 360-degree encircling scleral buckle was placed using standard techniques. The site of all breaks was identified with indirect ophthalmoscopy and scleral depression. The buckle was positioned to support the identified breaks and/or vitreous base. A \#42 band or \#41 band (Labtician, Oakville, Ontario, Canada) was sutured into position utilizing 5-0 nylon horizontal mattress sutures and the buckle was opposed with a Watzke sleeve (Labtician). The buckle was raised to a moderate height prior to proceeding with vitrectomy. Simultaneous clear corneal cataract surgery was performed by the vitreoretinal surgeon using standard phacoemulsification techniques in three of the cases. A 10-0 nylon suture was placed in the corneal incision at the conclusion of the cataract surgery.

All PPV and cataract surgery was performed utilizing the Accurus ${ }^{\circledR}$ Vitreoretinal Surgical System (Alcon Laboratories Fort Worth, TX, USA) and either Xenon (Alcon) or Photon (Synergetics, 0'Fallon, MO, USA) light sources. The trocar/cannula system (Alcon) was used to place 23-gauge cannulas in the superonasal, superotemporal and inferotemporal quadrants. The trocar/cannula incisions were made in a beveled fashion through bare 
sclera. The conjunctiva had been previously recessed during the peritomy for scleral buckling. Cannulas were placed inferotemporally, superotemporally, and superonasally $3 \mathrm{~mm}$ posterior to the limbus for pseudophakic eyes and $4 \mathrm{~mm}$ for phakic eyes. The infusion line was connected to the inferotemporal cannula. Wide-angle fundus visualization was achieved using either the BIOM noncontact wide field imaging system (Oculus, Munich, Germany) or the AVI contact panoramic viewing system (Advanced Visual Systems Inc., NY, NY, USA).

The surgical procedures varied slightly depending upon the location and extent of the retinal detachment and the individual preferences of the four surgeons. Generally, a core vitrectomy was performed followed by meticulous peripheral vitreous dissection with particular attention to relieving all traction at the sites of retinal breaks and along detached retina. Scleral depression was performed when necessary. In many cases, Perfluoron (Alcon) was utilized to stabilize detached retina and facilitate egress of subretinal fluid through peripheral retinal breaks. Membrane peeling was performed using end-grasping forceps. Retinectomy was performed in some cases utilizing either the vitreous cutter or intraocular scissors. A fluid-air exchange with a soft tip extrusion needle with active suction or the vitreous cutter itself was used to drain subretinal and posterior pole fluid. A directional endolaser (Synergetics) and/or laser indirect ophthalmoscope was utilized to demarcate retinal breaks and in some cases to perform 360 degree laser or demarcate a retinectomy border. Nonexpansile mixtures of either perfluoropropane (C3F8) or sulfur hexafluoride (SF6) were used, and in many cases, 5000 centistoke silicone oil (Bausch and Lomb, San Dimas, CA, USA) was utilized for tamponade.

At the conclusion of each procedure, the sclerotomies were closed with 7-0 vicryl sutures for many of the patients. The overlying conjunctiva was closed with 6-0 plain gut suture. Subconjunctival antibiotic and dexamethasone was administered. Ciloxan ophthalmic ointment was placed on the surface of the eye followed by a patch and shield. Patients were instructed to position either face down or one side down for on average 7 days.

Patient medical records were reviewed. Age, gender, eye, ophthalmic history, pre- and post-operative Snellen visual acuities, surgery data, intra- and post-operative complications, final attachment rates and need for subsequent retinal detachment repair were obtained. Length of follow up was also recorded. A total of 54 eyes in 53 patients were identified. One male patient underwent surgery on both eyes during the course of the study; one eye had a RRD with high myopia and the other eye had a GRT. With the exception of patient gender, all statistical analysis and percentages were in reference to the number of eyes.

Snellen visual acuities were converted to $\log$ MAR values for statistical analysis using the following equation: $\log M A R=-\log$ (visual fraction). LogMAR acuities were converted back to Snellen visual acuities for reporting mean preoperative and postoperative visual acuities. For visual acuities worse than $20 / 400$, visual function was recorded as count fingers (CF), hand motion (HM) or light perception (LP). No patients had no light perception vision pre or postoperatively. LogMAR values were assigned as 2.0 (CF vision), 2.3 (HM vision) or 2.6 (LP vision), as has been used in other studies. ${ }^{19-20}$ A student's t-test with a $5 \%$ level of significance was used to determine if there was a statistical difference in the pre and postoperative logMAR visual acuities, and to compare postoperative complication rates among those with silicone filled eyes versus gas filled eyes.

\section{Results}

Patient demographics are summarized in Table 1 . There were 54 eyes of 53 patients who underwent 23GPPV/SB. Mean age of the 54 eyes was 53.96 years (range of 18-88 years, standard deviation of 15.33). Right eyes were affected in $48.1 \%$ (26 of 54 eyes), while left eyes were affected in $51.9 \%$ ( 28 of 54 eyes). 41 of 53 patients $(77.4 \%)$ were males, while the remaining 12 patients (22.6\%) were females. One male patient underwent surgery on both eyes during the study. The mean follow up time for each eye was 8.96 months (standard deviation 5.61, range 1 to 23 months).

The indications for surgery included the following: RRD with any grade of PVR (15 of 54 eyes, $27.8 \%$ ), multiple breaks associated with RRD (13 of 54 eyes, 24.1\%), GRT (9 of 54 eyes $16.7 \%$ ), high myopia (8 of 54 eyes, 14.8\%), trauma (4 of 54 eyes, 7.4\%), and other (5 of 54 eyes, $4.8 \%$ ). Lens status was as follows: $37.0 \%$ of eyes were phakic (20 of 54 eyes), $59.3 \%$ were pseudophakic (32 of 54 eyes), one was aphakic (1.9\%) and one had an anterior chamber intraocular lens (1.9\%). Fifteen of 54 eyes (27.8\%) had undergone a prior pars plana vitrectomy.

Table 2 summarizes surgical techniques and intraoperative variables. A 360-degree encircling scleral buckle was placed in all eyes. The number of eyes with simultaneous cataract extraction was three of 54 (5.6\%) and one eye underwent a retinectomy at the time of surgery. The most commonly used tamponading agent was SF6 $(61.1 \%, 33$ of 54 eyes), with silicone oil $(24.1 \%, 13$ of 54 eyes) and $\mathrm{C}_{3} \mathrm{~F}_{8}(14.8 \%, 8$ of 54 eyes) used less frequently. The vast majority of eyes had a 42 band placed (88.9\%, 48 of 54 eyes) as opposed to a 41 band $(11.1 \%, 6$ of 54 eyes). Perfluoron-octane (PFO) was used in $66.7 \%$ of eyes (36 of 54 eyes). All sclerotomies were left open in three eyes and a portion was left open in four eyes. All 23-gauge sclerotomy incisions were closed with a single interrupted 7-0 vicryl suture in the majority of cases (47 eyes).

Visual outcomes, anatomic success and

Table 1. Patient demographics.

\begin{tabular}{lc} 
Gender & Number of patients (\%) \\
Male & $41(77.4)^{*}$ \\
Female & $12(22.6)$ \\
Age of 54 eyes & \\
Mean & 53.96 years \\
Standard deviation & 15.33 \\
\hline
\end{tabular}

Follow up time of 54 eyes

Mean 8.96 months

Standard deviation 5.61

\begin{tabular}{lc} 
& Number of eyes (\%) \\
Eye involved & \\
Right & $26(48.1)$ \\
Left & $28(51.9)$ \\
Diagnosis & \\
RRD with PVR & $15(27.8)$ \\
Multiple tears & $13(24.1)$ \\
GRT & $9(16.7)$ \\
Myopia & $8(14.8)$ \\
Traumatic & $4(7.4)$ \\
Other & $5(4.8)$ \\
\hline Prior PPV & \\
Yes & $15(27.8)$ \\
No & $39(72.2)$ \\
Lens Status & \\
Phakic & $20(37.0)$ \\
Pseudophakic & $32(59.3)$ \\
ACIOL & $1(1.9)$ \\
Aphakic & $1(1.9)$ \\
\hline
\end{tabular}

*One male patient had surgery on both eyes. RRD, rhegmatogenous retinal detachment; PVR, proliferative vitreoretinopathy; GRT, giant retinal tear; $\mathrm{ACIOL}$, anterior chamber intraocular lens.

Table 2. Intraoperative features.

\begin{tabular}{|c|c|}
\hline & $\begin{array}{c}\text { Number of eyes } \\
\text { (\% of eyes) }\end{array}$ \\
\hline \multicolumn{2}{|l|}{ Additional step } \\
\hline Retinectomy & $1(1.9 \%)$ \\
\hline Cataract extraction & $3(5.6 \%)$ \\
\hline PFO & $36(66.7 \%)$ \\
\hline \multicolumn{2}{|l|}{ Intraocular tamponade } \\
\hline $\mathrm{SF}_{6}$ & $33(61.1 \%)$ \\
\hline $\mathrm{C}_{3} \mathrm{~F}_{8}$ & $8(14.8 \%)$ \\
\hline Silicone Oil & $13(24.1 \%)$ \\
\hline \multicolumn{2}{|l|}{ Band Placed } \\
\hline 41 & $6(11.1 \%)$ \\
\hline 42 & $48(88.9 \%)$ \\
\hline
\end{tabular}

PPV, pars plana vitrectomy; PFO, perfluoro-n-octane. 
complications are summarized in Table 3 . Mean $\operatorname{logMAR}$ preoperative visual acuity was 1.166 (20/293) with a standard deviation of 0.941 . Mean $\log M A R$ postoperative visual acuity was 0.780 (20/120) with a standard deviation of 0.685 . Visual acuity significantly improved by $0.386 \operatorname{logMAR}$ units $(\mathrm{P}=0.0165)$. The anatomic reattachment rate was $87.0 \%$ after one operation (47 of 54 eyes), with all eyes ultimately remaining attached. Seven eyes developed recurrent retinal detachment (rhegmatogenous and/or tractional) and required additional surgery. Six were due to PVR and one was related to a choroidal hemorrhage. Two eyes required a third retinal detachment repair.

Additional postoperative complications other than recurrent retinal detachment were common in this series reflecting the complexity of the underlying pathology. As listed in table 3 , complications were separated based on when they presented clinically: intraoperative/ immediate (postoperative day one) or delayed (postoperative day two or beyond). Choroidal effusion or hemorrhage occurred in 8 of 54 eyes (14.8\%). Five eyes had an intraoperative or immediate choroidal effusion or hemorrhage. Only one of the eight eyes with a choroidal effusion or hemorrhage had coexisting hypotony. Vitreous hemorrhage occurred in 6 eyes (11.1\%), of which two were immediate and resolved spontaneously. Three of four eyes with a delayed vitreous hemorrhage occurred in eyes that re-detached and required additional surgery. A hyphema occurred in five eyes (9.3\%). Delayed hyphemas (three of five eyes) resolved spontaneously, but the two eyes with immediate hyphemas also developed vitreous and suprachoroidal hemorrhages. Hypotony occurred in three of 54 eyes $(5.6 \%)$. It resolved spontaneously in two eyes, but one eye also developed a choroidal hemorrhage, hyphema, vitreous hemorrhage, and ultimately required a second retinal detachment repair. Ocular hypertension with an intraocular pressure greater than $35 \mathrm{mmHg}$ occurred on P0D1 in 2 of 54 eyes $(3.7 \%$ of eyes), but resolved by the next postoperative visit. A total of 17 eyes (31.5\%) had one of these complications at some point during the intraoperative or postoperative course, but over half of these (59.9\%) resolved without any intervention. Two patients had at least four complications, including hyphema, vitreous hemorrhage, choroidal hemorrhage and a retinal detachment. They attained final Snellen visual acuities of 20/200 and 20/400. There was a nonsignificant trend towards decreased complications in silicone filled eyes (15.4\% versus $36.6 \%$ in gas filled eyes, $\mathrm{P}=0.157$ ).

\section{Discussion}

Prior studies have described complex rhegmatogenous retinal detachments as those that have included PVR, GRT, ocular trauma, high/pathologic myopia, PDR, and necrotizing retinitis ${ }^{11}$ Surgeon preference influences the surgical approach to more complex pathology. Many surgeons would consider combined scleral buckling and 20-gauge PPV for these cases. ${ }^{15-18,21-24}$

A recent trend in vitreoretinal surgery has been the application of smaller gauge vitrectomy instruments for increasing complex retinal pathologies. Twenty three-gauge PPV has been shown to be associated with more rapid visual recovery than traditional 20 -gauge PPV in epiretinal membrane surgery. ${ }^{25}$ Others have used 23-gauge PPV for a multitude of posterior segment pathology with few complications and improved visual acuity. ${ }^{26-28}$ Given the recent literature supporting the use of smaller vitrectomy instruments with a good visual outcome and safety profile, the goal of the current review was to describe results using 23-gauge pars plana vitrectomy in combination with scleral buckling in these complex retinal detachment repairs.

The main outcome measures included preoperative and postoperative visual acuities, postoperative complications, and anatomic success. Visual acuities were significantly better postoperatively. Preoperative Snellen acuities averaged about 20/300 and postoperatively were about 20/120. The role of induced corneal astigmatism by surgery was not addressed in this study, but prior studies have shown this to be less of an issue in those undergoing 23-gauge PPV as compared to 20gauge PPV. ${ }^{25}$ Surgically induced corneal astigmatism is unlikely to be a critical factor in patients with complex RRDs and generally poor postoperative visual acuity results. However, a large proportion of patients with complex rhegmatogenous RRD do achieve good visual acuities and PPV-induced astigmatism may be a factor in their visual rehabilitation. Immediate and delayed complications were not uncommon, as nearly one third of patients had a hyphema, ocular hypertension or hypotony, choroidal effusion or hemorrhage, vitreous hemorrhage or retinal detachment at some point in the postoperative period. The most common complications were choroidal effusions/hemorrhages and vitreous hemorrhages. One hypothesis is that there may be transient hypotony postoperatively in these cases. This may occur even when 23-gauge sclerotomy sites are closed with 7-0 vicryl sutures. The trocar system used in this series produces an irregular $t$-shaped sclerotomy site even when made in a beveled fashion and thus may not close completely as with a linear 20-
Table 3. Visual outcomes and complications.

\begin{tabular}{lc} 
Mean preoperative visual acuity & \\
Snellen & $20 / 293$ \\
LogMAR & 1.166 \\
Mean postoperative visual acuity & \\
Snellen & $20 / 120$ \\
LogMar & 0.780 \\
LogMAR improvement & 0.386 \\
& Number of eyes \\
& $(\%$ of eyes) \\
Anatomic Success & \\
After one surgery & $47(87.0)$ \\
After two surgeries & $52(96.3 \%)$ \\
Final & $54(100 \%)$ \\
Immediate or intraoperative & \\
complications & \\
Ocular hypertension & $2(3.7 \%)$ \\
Hypotony & $1(1.9 \%)$ \\
Hyphema & $2(3.7 \%)$ \\
Vitreous hemorrhage & $2(3.7 \%)$ \\
Choroidal effusion/hemorrhage & $5(9.3 \%)$ \\
\hline
\end{tabular}

Delayed complications

(Prestenting POD2 and beyond)

\begin{tabular}{lr} 
Ocular hypertension & $0(0.0 \%)$ \\
Hypotony & $2(3.8 \%)$ \\
Hyphema & $3(5.6 \%)$ \\
Vitreous hemorrhage & $4(7.4 \%)$ \\
Choroidal effusion/hemorrhage & $3(5.6 \%)$ \\
Number of eyes & $17(31.5 \%)$ \\
\hline
\end{tabular}

with any complication

VA, visual acuity; logMAR, logarithm of the minimal angle of resolution; POD2, postoperative day 2.

gauge incision. Several patients also had other risk factors for transient hypotony, including high myopia and prior pars plana vitrectomy. Overall, postoperative complications (with the exception of recurrent retinal detachment) were generally self-limited and did not affect final anatomic result or visual acuity results.

The complication rates reported in this study are higher than those previously published by others for 20 gauge PPV with scleral buckling. Wickham et al. ${ }^{22}$ and Gartry et al. ${ }^{14}$ reported vitreous hemorrhage in approximately $5-7 \%$ and choroidal hemorrhage in $4 \%$ of patients undergoing combined 20-gauge PPV with scleral buckling. However, the inclusion and exclusion criteria were different from the current review. In the former, ${ }^{22}$ patients were excluded if they had prior PPV, had grade $\mathrm{C}$ or greater PVR or GRTs. The latter article ${ }^{14}$ reviewed cases of relatively uncomplicated RRD, excluding GRTs and more advanced PVR. Albrieux et al. compared 23-gauge PPV to 20 gauge PPV for RRD repair. ${ }^{29}$ They found a similar rate of postoperative complications and anatomic reattachment among the two groups. While the rate of complications was lower than the current review (one choroidal detachment in the 23 gauge group, no hypotony, no reported vitreous hemorrhage or hyphema), SBP was not performed, and patients were excluded if 
they had prior PPV, traumatic RD, PVR grade C, GRTs, among other exclusion criteria.

In the current review, patients had an anatomic reattachment rate of $87 \%$ after one surgery, but reached final reattachment in all cases. The reattachment rates in this study were similar to those reported by others for more complex retinal detachments. ${ }^{10,12,14,15,22}$ Single operation reattachment rates were lower than those reported by Stangos et al. (92.3\%) and Weichel et al. (94\%), but these patients underwent 20 gauge PPV with SBP for pseudophakic RRDs without associated PVR or GRT. ${ }^{13,28}$ The anatomic reattachment reported by Albrieux et al. for PPV without SBP was $74.3 \%$ (20 gauge group) and 80\% (23 gauge group). ${ }^{29}$ The anatomic reattachment rate in the current review was similar to another study comparing 20 (89.3\%), 23 (88.9\%) and 25 gauge PPV (93.3\%) without SBP for pseudophakic RRDs in patients without prior PPV. ${ }^{30}$

There are significant limitations to this retrospective study. First, the sample size is fairly small and indications for surgery were variable. Also, there are a large number of confounding variables that were present, including a history of prior PPV, gas or silicone used for intraocular tamponade, use of PFO, closure of sclerotomies and simultaneous cataract extraction or retinectomy. Certainly, some of these variables might play a role in the single operation reattachment rates and the development of postoperative complications.

The potential advantages of 23GPPV/SB surgery versus 20 -gauge includes smaller sclerotomy size, cannula based infusion line placement (versus suture fixation), and possibly reduced astigmatism. In the past, smaller gauge surgeries for more complex detachments have been limited due to reduced instrument rigidity and a reduced armamentarium of instruments (e.g. scissors). However, a wider array of ancillary instruments is available and 23-gauge PPV instruments have significant rigidity allowing complete eye rotation/control and dissection of dense fibrovascular tissue. Recent studies have shown similar flow rates between 23 - and 20 -gauge instruments thus enabling high vacuum based maneuvers. ${ }^{31,32}$ Eventually, when a 23 -gauge fragmotome for lensectomy is produced, the 23-gauge platform may replace 20 gauge PPV in most cases.

In summary, we conclude that 23-gauge pars plana vitrectomy with scleral buckling is a viable alternative to the traditional 20 -gauge pars plana vitrectomy with scleral buckling. Our retrospective study shows a significant improvement in visual acuity after surgery, with a high single operation reattachment rate and a final reattachment rate of $100 \%$. However, our study did show that postoperative complications (especially vitreous hemorrhage and choroidal effusion/hemorrhage) are higher than those reported in some prior studies ${ }^{14,22}$ and that these complications need to be further studied. It is unknown whether the higher complication rate is related to the 23 gauge platform or a difference in patient population, with more complex PVR, ocular trauma, and GRTs included in the current review. Ideally, a study to compare traditional 20 -gauge PPV with scleral buckling and 23-gauge PPV with scleral buckling would help elucidate whether or not these complications are related to smaller gauge vitrectomy instruments and incisions, or related to the highly complex nature of retinal detachment repair in these patients.

\section{References}

1. Brazitikos PD, D’Amico DJ, Tsinopoulos IT, Stangos NT. Primary vitrectomy with perfluoro-n-octane use in the treatment of pseudophakic retinal detachment with undetected retinal breaks. Retina 1999;19: 103-9.

2. McCuen BW 2nd, Landers MB 3rd, Machemer R. The use of silicone oil following failed vitrectomy for retinal detachment for advanced proliferative vitreoretinopathy. Ophthalmology 1985;92:102934.

3. Lesnoni G, Billi B, Rossi T, Stirpe M. The use of panoramic viewing system in relaxing retinotomy and retinectomy. Retina 1997;17:186-90.

4. Lincoff $\mathrm{H}$, Coleman J, Kreissig I, et al. The perfluorocarbon gases in the treatment of retinal detachment. 0phthalmology 1983;90:546-51.

5. Fujii GY, de Juan E Jr, Humayun MS, et al. Initial experience using the transconjunctival sutureless vitrectomy system for vitreoretinal surgery. Ophthalmology 2002; 109:1814-20.

6. Lakhanpal RR, Humayun MS, de Juan E Jr, et al. Outcomes of 140 consecutive cases of 25-gauge transconjunctival surgery for posterior segment disease. Ophthalmology 2005;112:817-24.

7. Ibarra MS, Hermel M, Prenner JL, Hassan TS. Long-term outcomes of transconjunctival sutureless 25-gauge vitrectomy. Am J Ophthalmol 2005;139:831-6.

8. Miller DM, Riemann CD, Foster RE, Peterson MR. Primary repair of retinal detachment with 25 -gauge pars plana vitrectomy. Retina 2008;28:931-6.

9. Shah CP, Ho AC, Regillo CD, et al. Shortterm outcomes of 25-gauge vitrectomy with silicone oil for repair of complicated retinal detachment. Retina 2008;28:723-8. 10. Misra A, Ho-Yen G, Burton RL. 23-gauge sutureless vitrectomy and 20-gauge vitrectomy: a case series comparison. Eye (Lond) 2009;23:1187-91.

11. Azen SP, Scott IU, Flynn HW Jr, et al. Silicone oil in the repair of complex retinal detachments: A prospective observational multicenter study. Ophthalmology 1998; 105: 1587-97.

12. Sirimaharaj M, Balachandran C, Chan WC, et al. Vitrectomy with short term postoperative tamponade using perfluorocarbon liquid for giant retinal tears. Br J Ophthalmol 2005;89:1176-9.

13. Weichel ED, Martidis A, Fineman MS, et al. Pars plana vitrectomy versus combined pars plana vitrectomy-scleral buckle for primary repair of pseudophakic retinal detachment. Ophthalmology 2006;113: 2033-40.

14. Gartry DS, Chignell AH, Franks WA, Wong D. Pars plana vitrectomy for the treatment of rhegmatogenous retinal detachment uncomplicated by advanced proliferative vitreoretinopathy. Br J Ophthalmol 1993; 77:199-203.

15. Han DP, Pulido JS, Mieler WF, Johnson MW. Vitrectomy for proliferative diabetic retinopathy with severe equatorial fibrovascular proliferation. Am J Ophthalmol 2005;119:563-70.

16. Sigueira RC, Gomes CV, Dalloul C, Jorge R. Vitrectomy with and without scleral buckling for retinal detachment. Arq Bras Oftalmol 2007;70:298-302.

17. Boscia F, Furino C, Recchimurzo N, et al. Oxane HD vs silicone oil and scleral buckle in retinal detachment with proliferative vitreoretinopathy and inferior retinal breaks. Graefes Arch Clin Exp Ophthalmol 2008;246:943-8.

18. Goezinne F, La Heij EC, Berendschot T, et al. Low redetachment rate due to encircling scleral buckle in giant retinal tears treated with vitrectomy and silicone oil. Retina 2008;28:485-92.

19. Arevalo JF, Garcia RA, Wu L, et al. Radial optic neurotomy for central retinal vein occlusion: results of the Pan-American Collaborative Retina Study Group (PACORES). Retina 2008;28:1044-52.

20. Optic nerve decompression surgery for nonarteritic anterior ischemic optic neuropathy (NAION) is not effective and may be harmful. The ischemic optic neuropathy decompression trial research group. JAMA 1995;273:625-32.

21. Alexander P, Ang A, Poulson A, Snead MP. Scleral buckling combined with vitrectomy for the management of rhegmatogenous retinal detachment associated with inferior retinal breaks. Eye 2008;22:200-3.

22. Wickham L, Connor M, Aylward GW. Vitrectomy and gas for inferior break retinal detachments: are the results compara- 
ble to vitrectomy, gas and sclera buckle? $\mathrm{Br}$ J Ophthalmol 2004;88:1376-9.

23. Sharma A, Grigoropoulos V, Williamson TH. Management of primary rhegmatogenous retinal detachments with inferior breaks. Br J Ophthalmol 2004;88:1372-5.

24. Scott IU, Murray TG, Flynn HW Jr, et al. Outcomes and complications associated with giant retinal tear management using perfluoro-n-octane. Ophthalmology 2002; 109:1828-33.

25. Hikichi T, Matsumoto N, Ohtsuka H, et al. Comparison of one-year outcomes between 23 - and 20-gauge vitrectomy for preretinal membrane. Am J Ophthalmol 2009;147:639-43.el.

26. Gupta OP, Ho AC, Kaiser PK, et al. Short- term outcomes of 23-gauge pars plana vitrectomy. Am J Ophthalmol 2008;146:193-7.

27. Fine HF, Iranmanesh R, Iturralde D, Spaide RF. Outcomes of 77 consecutive cases of 23-gauge transconjunctival vitrectomy surgery for posterior segment disease. Ophthalmology 2007;114:1197-200.

28. Stangos AN, Petropoulos IK, Brozou CG, et al. Pars-plana vitrectomy alone vs vitrectomy with scleral buckling for primary rhegmatogenous pseudophakic retinal detachment. Am J Ophthalmol 2004;138:952-8.

29. Albrieux M, Rouberol F, Bernheim D, et al. Comparative study of 23-gauge vitrectomy versus 20 -gauge vitrectomy for the treatment of rhegmatogenous retinal detachment. Graefes Arch Clin Exp Ophthalmol
2011 Apr 16. [Epub ahead of print]

30. Lewis SA, Miller DM, Riemann CD, et al. Comparison of 20-, 23-, and 25-gauge pars plana vitrectomy in pseudophakic rhegmatogenous retinal detachment repair. Ophthalmic Surg Lasers Imaging 2011; 42:107-13.

31. Hubschman JP, Gupta A, Bourla DH, et al. 20-, 23-, and 25-gauge vitreous cutters: performance and characteristics evaluation. Retina 2008;28:249-57.

32. Magalhaes $0 \mathrm{Jr}$, Chong L, DeBoer C, et al. Vitreous dynamics: vitreous flow analysis in 20-, 23-, and 25-gauge cutters. Retina 2008;28:236-41. 